\title{
Analytical Modeling of a Traffic Differentiation Strategy for 802.11 \\ (Extended Abstract)
}

\author{
Luca Vollero and Giulio Iannello \\ Federico II University, Napoli, Italy, \\ DIS, Dipartimento di Informatica e Sistemistica, \\ \{vollero, iannello\}@unina.it
}

\section{Introduction}

Wireless communications are going to be the common way for individuals to connect with other people and to access Internet services. However, in spite of the many advantages exhibited by wireless technologies, they still have a number of drawbacks, such as poor reliability, limited throughput, scarce QoS and security support, limiting a wider applicability. An area that deserves attention is concerned with flexible mechanisms to guarantee and control the access to the communication medium under loaded conditions. Indeed, these mechanisms may represent the basis, for instance, to provide QoS guarantees to multimedia and other real-time applications, or to enforce protection from denial-of-service attacks.

In this paper we propose a modification to the MAC layer of the IEEE 802.11 standard to handle traffic differentiation in the so-called infrastructure configurations ([1], section 3.8). These networks of 802.11 compliant devices are characterized by multiple mobile stations, acting as terminal devices, that communicate through one or more base stations, acting as bridges for the wireless connections. Our proposal is based on introducing a selective acknowledgment schema at the base station side, which differentiates the network congestion status perceived by the mobile stations. In other words, bandwidth allocation is controlled by changing the reaction of the back-off algorithm to messages sent from stations belonging to different classes. The key aspect of our approach is that it requires modifications only to the base station, and it can be implemented at firmware level without changes at the physical layer. No change is required to the mobile stations, making the approach feasible in practice with limited effort.

To evaluate the effectiveness of the approach we extended the probabilistic model introduced by Bianchi [3] to evaluate the throughput of the 802.11 MAC layer in saturation conditions, and we used it to analyze the behavior of a modified base station with two classes of mobile stations. The analysis reveals that the traffic differentiation mechanism is effective in protecting bandwidth resources assigned to the privileged class under a wide range of situations. 


\section{The Modified MAC}

The Distributed Coordination function (DCF) is the basic IEEE 802.11 MAC mechanism. It is a distributed algorithm for the wireless channel access, based on a CSMA/CA (Carrier Sense Multiple Access with Collision Avoidance) schema.

Before accessing the medium, the generic transmitting station senses the channel to avoid collision with other stations and waits until the channel is idle. When the channel becomes idle, it waits for a DCF Interframe Space, DIFS, and then starts a back-off timer to randomize the starting transmitting time. Finally, when the timer expires, the station starts the transmission.

All transmissions are acknowledged. The receiver, immediately after data frame reception, waits for a Short Interframe Space (SIFS), and then sends back an ACK frame to the transmitter. Based on the acknowledgment status, the stations change adaptively their maximum transmitting frame rate, in order to reduce the probability that two or more transmitting stations collide.

The original version of DCF supports a form of traffic differentiation relying upon a polling schema, called Point Coordination Function (PCF). In [5], the combination of DCF and PCF was proved to limit both the maximum achievable throughput and the maximum payload size that can be transmitted. Hence, to introduce better traffic differentiation mechanisms in the IEEE 802.11, it is mandatory a modification to the standard MAC protocol. As discussed in section 5. previous proposals focused on the change to the back-off behavior and on its combination with the IFSs ([7], [10], and [11]).

While these approaches may be effective in the general case, we believe they may be improved for infrastructure configurations, i.e. networks of 802.11 devices characterized by the presence of one or more base stations (BSs), connected to the wired network and acting as bridges for wireless communications, and multiple mobile stations (MSs), acting as terminal devices.

The inherent asymmetric organization of infrastructure configurations can be exploited to minimize the MAC modifications required to support traffic differentiation. The approach we propose is to modify only the BS acknowledgment behavior to differentiate the network congestion status perceived by the mobile stations, saving all the other characteristics of the standard. The idea behind this proposal is that the throughput of the generic MS can be limited through this simple selective acknowledgment mechanism, allowing for a centralized control of traffic differentiation through congestion simulation.

In practice, with two traffic classes, the mechanism can be implemented by introducing a dropping factor $d_{f}$ for the lower priority class over a control window of $L$ received frames. Dropping $l=d_{f} \cdot L$ frames every $L$ frames received from lower priority stations, causes DCF to reduce the throughput of the low priority class in order to adapt to the higher perceived congestion status.

This approach leads up to a number of advantages. First, the exploitation of the inherent asymmetric behavior of BS configurations allows us to limit MAC layer modifications to just one station. Second, the selective acknowledgment can be implemented at the firmware level without hardware changes. Third, the ability to control the station resources through a centralized mechanism allows us 
to verify continuously the total amount of available bandwidth and to easy define mechanisms for the stations admission control and resources redistribution.

\section{The Analytical Model}

In [3], Bianchi presents an analytical model for the evaluation of the IEEE 802.11 LAN throughput in saturation conditions. The model assumes that the system reaches a stable state where the frame collision probability can be supposed constant and independent of other parameters. The mathematical results derived under these hypotheses are compared with performance data produced through simulations, confirming the effectiveness of the approach.

As a first step to evaluate the effectiveness of the proposed modified MAC, we choose to follow a similar analytical approach and extended the Bianchi's model to develop an analysis in saturation conditions.

To make the reading of the following formulas easier, we have summarized in table 1 all symbols used by our extended model.

Table 1. Symbols used in the formulas.

\begin{tabular}{|c|c|}
\hline Name & Description \\
\hline \hline$m$ & maximum retransmission back-off \\
\hline$W$ & minimum back-off window \\
\hline$n_{i}$ & number of transmitting stations in class $i$ \\
\hline$\tau_{i}$ & probability that a generic station in class $i$ transmits on a given time slot \\
\hline$p$ & probability that a given frame collides with other transmitted frames \\
\hline$\Delta$ & additional collision probability perceived by low priority stations \\
\hline
\end{tabular}

To maintain the model as simple as possible, we limited our analysis to the case of two transmission classes, referred to in the following as class 1 (higher priority) and class 2 (lower priority), respectively.

The key approximation in the Bianchi's model is that each packet collides with constant and independent probability $p$. Our schema uses the same approximation and modifies the model introducing two transmission classes. Since our differentiation technique corresponds to different collision probabilities, in the extended model transmission and collision probabilities are mutually related according to the following set of equations:

$$
\begin{aligned}
\tau_{1} & =\frac{2(1-2 p)}{(1-2 p)(W+1)+p W\left(1-(2 p)^{m}\right)} \\
\tau_{2} & =\frac{2(1-2 \tilde{p})}{(1-2 \tilde{p})(W+1)+\tilde{p} W\left(1-(2 \tilde{p})^{m}\right)}
\end{aligned}
$$




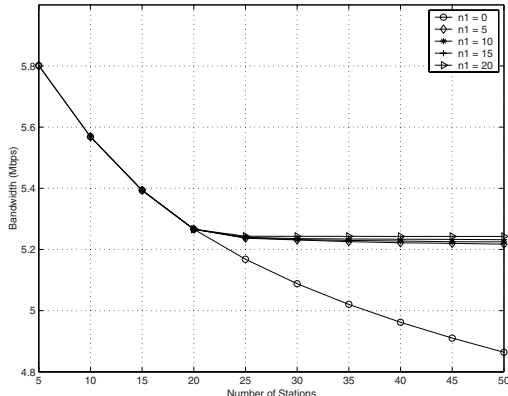

Fig. 1. Total bandwidth utilization.

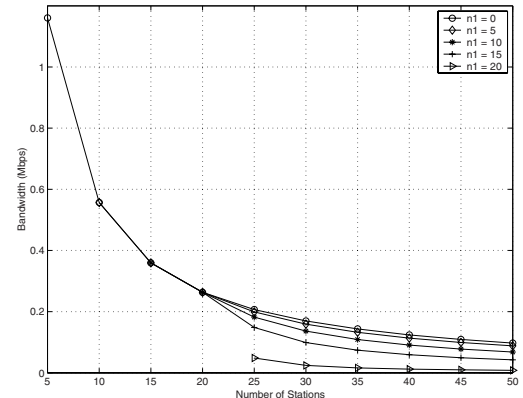

Fig. 2. Bandwidth utilization of each station in class 2 .

$$
\begin{gathered}
p=1-\left(1-\tau_{1}\right)^{n_{1}-1}\left(1-\tau_{2}\right)^{n_{2}} \\
\tilde{p}=1-\left(1-\tau_{1}\right)^{n_{1}}\left(1-\tau_{2}\right)^{n_{2}-1}+\Delta
\end{gathered}
$$

where $\tau_{1}$ and $\tau_{2}$ are the transmission probabilities, $p$ and $\tilde{p}$ are the collision probabilities, and $n_{1}$ and $n_{2}$ are the number of transmitting stations for traffic classes 1 and 2, respectively, and $\Delta$ is the additional collision probability induced by the modified MAC for traffic class 2 .

Given $\Delta$, the above extended model can be solved, obtaining the corresponding transmission and collision probabilities of the two classes (see 44). With these parameters we can derive both the dropping factor $d_{f}$ the BS has to apply to achieve the desired traffic differentiation, and the normalized saturation throughput of the two classes, which represents the primary performance index to evaluate the selective acknowledgment strategy.

\section{Performance Characterization}

In this section, we report the results obtained from the model presented above. We evaluate the behavior of a set of MSs partitioned into two traffic classes, varying both the total number of stations and the number of stations in class 1 . We developed the analysis using a constant frame length of 1 Kbytes and a constant assured throughput of $250 \mathrm{Kbps}$ for each MS in the privileged class. These parameters could be also varied, but for the sake of simplicity we maintained them constant (for a more detailed analysis, see 44). For the model parameters we used the typical values of the 802.11 b standard.

Total bandwidth in saturation conditions vs. the number of MSs is reported in figure 1. Different curves refer to a different number of class $1 \mathrm{MSs}$. The curve corresponding to $n_{1}=0$ represents the behavior of standard DCF according to 
what estimated by Bianchi's model (the curve presented here differs from the one reported in [3] because a transmission rate of $1 \mathrm{Mbps}$ is assumed in the cited article). Graphs show that the use of our differentiation technique leads to a reduction of unused bandwidth when the total number of MSs overcomes a given threshold.

The bandwidth of a single MS in class 2 is reported in figure 2. It shows that our mechanism is able to differentiate traffic effectively avoiding complete starvation of stations in low priority class.

\section{Related and Future Work}

In the literature, a lot of work has been done on the problem of distributed access mechanisms supporting traffic differentiation and throughput enhancement.

In [5], Visser and Zarki present simulation results for a combination of speech and data communications over a IEEE 802.11 LAN, using PCF the polling schema provided from the 802.11 standard. In [6], Sobrinho and Krishnakumar present a distributed solution supporting real-time transmissions, specialized only for isochronous sources. In [7], Deng and Chan propose a method to support station priority, changing both the interframe space (IFS) used between data frames, and the back-off mechanism.

In [10, Vaidya et al. present an access schema relying upon an adaptationd of fair queuing ([8], [9]) to the wireless environment. In [11], Banchs and Pérez propose an extension of the IEEE 802.11 MAC protocol referred to as ARME (Assured Rate MAC Extension).

Finally, motivated by the growing interest in wireless networks supporting QoS, the IEEE 802.11 Working Group started an activity to enhance the 802.11 MAC protocol. IEEE 802.11 Task Group E is currently defining enhancements to the basic 802.11 MAC, called 802.11e ([2]). The effort aims to introduce and extend the mechanisms proposed in the literature, and in particular those based on: (i) back-off and interframe space differentiation, and (ii) multiple independent back-off instances for each MS.

While our approach, being limited to infrastructure configurations, is less general of most of the above mentioned proposal, its implementation requires very limited changes only on the base station side.

Another interesting aspect of our approach is the ability to enforce absolute guarantees. Indeed, simply varying the simulated congestion, it is possible to increase or reduce the level of traffic differentiation, or, alternatively, adapt the current level to meet specific minimal requirements. Other solutions, like those discussed in 7] and [10], do not specify how to accomplish the latter task and how the differentiation level can be controlled easily.

Finally, as stressed by the performance analysis, our mechanism allows a fine grained control over resource assignments and it leads to improved overall channel utilization.

Future works will be mainly focused on validation of the used analytical model and on characterization of system dynamic behavior by simulations. A 
modified version of berkeley network simulator ([12]) has been already developed to achieve both these evaluations. Another future work is the extension of the analytical model to more general and complex cases, such as considering more than two traffic classes or RTS/CTS and hybrid MAC protocols.

Acknowledgements. This work has been carried out under the financial support of the Ministero dell'Istruzione, dell'Università e della Ricerca (MIUR) in the framework of the FIRB project "Middleware for advanced services over largescale, wired-wireless distributed systems (WEB-MINDS)", and of the project "Scalability and Quality of Service in Web Systems".

\section{References}

1. IEEE 802.11 WG, "Reference number ISO/IEC 8802-11: 1999(E) IEEE Std 802.11, 1999 edition. International Standard [for] Information TechnologyTelecommunications and Information exchange between system-Local and metropolitan area networks-Specific Requirements-Part 11: Wireless LAN Medium Access Control (MAC) and Physical Layer (PHY) specifications",1999.

2. IEEE 802.11 WG, Draft Supplement to STANDARD FOR Telecommunications and Information Exchange Between Systems - LAN/MAN Specific Requirements Part 11: Wireless Medium Access Control (MAC) and Physical layer (PHY) specifications: Medium Access Control (MAC) Enhancements for Quality of Services (QoS), IEEE 802.11e/D2.0, Nov. 2001.

3. G. Bianchi, Performance Analysis of the 802.11 Distributed Coordination Function. IEEE Journal on Selected Area in Comm. V18, N3, March 2000.

4. L. Vollero and G. Iannello, Analytical Modeling of a Traffic Differentiation Strategy for 802.11, Tech. Rep., Computer Science Department, Federico II University, Napoli, Italy, July 2003

5. M. A. Visser and M. El Zarki, Voice and data transmission over an 802.11 wireless network. In Proceedings of PIMRC'95, Toronto, Canada, pp. 648-652, September 1995.

6. J. L. Sobrinho and A. S. Krishnakumar, Real-time traffic over the IEEE 802.11 medium access control layer. Bell Labs Technical Journal, pp. 172-187, Autumn 1996.

7. D-J. Deng and R-S. Chang, A priority scheme for IEEE 802.11 DCF access method. IEICE Transactions on Communications, VE82-B, pp. 96-102 January 1999.

8. S. J. Golestani, A self-clocked fair queueing scheme for broadband applications. In Proceedings of IEEE INFOCOM, 1994.

9. P. Goyal, H. M. Vin, and Cheng, Start-time fair queueing: A scheduling algorithm for integrated services packet switching networks. IEEE/ACM Transactions on Networking, 5:690-705, October 1997.

10. N. H. Vaidya, P. Bahl, and S. Gupta. Distributed fair scheduling in a wireless LAN. In Proceedings of ACM MOBICOM 2000, Boston, MA, August 2000.

11. A. Banches and X. Pérez, Providing throughput guarantees in IEEE 802.11 wireless LAN. In Proceedings of WCNC, Vol. 1, pp. 130-138, March 2002.

12. NS2, The Network Simulator; http://www.isi.edu/nsnam/ns/ 